\title{
Umbilical Cord Blood-Derived Mesenchymal Stem Cells Transplantation Decreases Incidence of Liver Cancer in End-Stage Liver Disease Patients: A Retrospective Analysis Over 5 Years
}

\section{Le Luo ( $\sim 2833859575 @ q q . c o m$ )}

Sichuan Provincial People's Hospital: Sichuan Academy of Medical Sciences and Sichuan People's Hospital https://orcid.org/0000-0002-8533-8427

\section{Cun-You Lai}

Sichuan Provincial People's Hospital: Sichuan Academy of Medical Sciences and Sichuan People's Hospital

\section{Tian-Hang Feng}

Sichuan Provincial People's Hospital: Sichuan Academy of Medical Sciences and Sichuan People's Hospital

\section{Yu-Tong Yao}

Sichuan Provincial People's Hospital: Sichuan Academy of Medical Sciences and Sichuan People's Hospital

\section{Hua Xue}

Sichuan Provincial People's Hospital: Sichuan Academy of Medical Sciences and Sichuan People's Hospital

\section{Guang-Ming Xiang}

Sichuan Provincial People's Hospital: Sichuan Academy of Medical Sciences and Sichuan People's Hospital

Hai-Bo Zou

Sichuan Provincial People's Hospital: Sichuan Academy of Medical Sciences and Sichuan People's Hospital

\section{Lan-Yun Luo}

Sichuan Provincial People's Hospital: Sichuan Academy of Medical Sciences and Sichuan People's Hospital

\section{Guan Wang}

Sichuan Provincial People's Hospital: Sichuan Academy of Medical Sciences and Sichuan People's Hospital

\section{Xiao-Lun Huang}

Sichuan Provincial People's Hospital: Sichuan Academy of Medical Sciences and Sichuan People's Hospital 


\section{Research}

Keywords: Umbilical cord blood-derived mesenchymal stem cells, transplantation, liver cancer, end-stage liver disease patients, retrospective analysis

Posted Date: August 24th, 2021

DOl: https://doi.org/10.21203/rs.3.rs-749694/v1

License: (c) (i) This work is licensed under a Creative Commons Attribution 4.0 International License. Read Full License 


\section{Abstract}

Background: End-stage liver disease (ESLD) is the final stage of a liver disease, which is characterized by liver cirrhosis. ESLD largely increases possibility of liver cancers in the world. The stem cell transplantation has become an emerging therapy to treat various liver diseases including ESLD, while whether it causes liver cancer remains unclear. The study aims to analysis the long-term therapeutic effect of umbilical cord blood-derived mesenchymal stem cells (UC-MSCs) transplantation in ESLD patients.

Patients and Methods: 50 ELSD patients of non-UC-MSCs transplantation and 45 ELSD patients of UCMSCs transplantation were retrospectively analyzed. The clinical outcomes in clinical and biochemical data, complications, and quality of life were recorded at $3,6,12,36$, and 60 months.

Results: It was found that the incidence of liver cancer was much lower in ESLD patients with UC-MSCs transplantation than in ESLD patients without UC-MSCs transplantation (12\% VS 2.2\%). The survival percentage was improved by treatment with UC-MSCs transplantation compared with non-UC-MSCs transplantation in ESLD patients during the five years follow-up. The inflammation and fibrosis scores were decreased in the ESLD patients with UC-MSCs transplantation. The liver cirrhosis was largely improved, and Child-Pugh scores were decreased.

Conclusions: UC-MSCs transplantation is able to decrease the risks of liver cancers in ELSD patients. The reduction of liver cancer incidence might be related to decrease of inflammation by UC-MSCs transplantation. More efforts should be investigated towards increasing the number of patients and follow-up time to further verify the therapeutic benefits of UC-MSCs translations in treating liver cancers.

\section{Introduction}

End-stage liver disease (ESLD) is the final stage of a liver disease, which largely increases possibility of liver cancers and results in high morbidity and mortality in the world (1). ESLD is characterized by the cirrhosis (1) and the age of patients with ESLD tend to younger people (2). Several etiologies including alcohol abuse, infections of chronic hepatitis $B$ virus (HBV) and hepatitis $C$ virus, and non-alcoholic steatohepatitis (NASH) are involved in liver cirrhosis (3), which often shows fibrosis and a profound chronic systemic inflammatory response (4). An inappropriate therapeutic method of inflammation may cause a variety of complications in liver cirrhosis patients (4). The conventional therapies including radiotherapies, chemotherapies, and anti-cancer drugs based conservative treatment against liver cirrhosis are not satisfied to heal the disease and harbor high risks to develop into liver cancer (5). Orthotopic liver transplantation is thought to be an effective therapy to treat liver cirrhosis, while which is limited to broadly use due to immunological rejection and the scarcity of donor sources (6). Therefore, a more effective and safe strategy against liver cirrhosis should be investigated to reduce severity of the disease. 
Accumulating studies indicated that long-term inflammation and fibrosis in the liver might be the reason that liver cirrhosis causing liver cancer $(7,8)$. Thus, suppression of inflammation and fibrosis might be one of solutions to reduce the risks of liver cancer. Stem cell transplantation was found to remarkably reduce the severity of inflammation and fibrosis (1). Stem cell transplantation technology was established in the 1950s (9). Each year, numerous stem cell transplantation cases were reported globally (10). It has been demonstrated that stem cell transplantation is an effective strategy in treating a variety of diseases including severe and refractory autoimmune diseases (AD) (11), non-rhabdomyosarcoma soft tissue sarcomas (12), kidney diseases (13), and multiple sclerosis and immune-mediated neurological diseases (14). Distinct types of stem or progenitor cells were reported to be used in transplantation, among, the hematopoietic stem cells (HSC) derived from peripheral-blood those are marked with CD34 and umbilical cord blood-derived mesenchymal stem cells (UC-MSCs) were most often used (15). The potential utilization of stem cells transplantation to treat various diseases is attracted more and more attention.

Emerging evidence indicate that transplantation of stem cells including HSCs and MSCs is an effective strategy to treat liver cirrhosis (6). It was demonstrated that treatment with stem cell transplantation in liver cirrhosis patients showed promising results, for example, the autologous infusion of CD34 + cells improved the serum albumin level and the Child-Pugh score in ELSD patients (6). However, studies using MSCs to treat liver cirrhosis and long-term follow-up outcomes post transplantation remain very limited. Moreover, whether MSCs transplantation could result in liver cancer remains unclear. Thus, in the present study, a retrospective analysis was performed towards the 5-year survival of the patients with ESLD that underwent UC-MSCs transplantation to figure out effects of UC-MSCs transplantation on liver cirrhosis and liver cancer incidence post transplantation. The findings in the study should provide crucial views for understanding the role of stem cell transplantation in treatment of liver cancer.

\section{Materials And Methods}

\section{Patient selection and methods}

The ESLD patients treated with UC-MSCs and control group (non-UC-MSCs transplantation) were retrospectively analyzed at Sichuan Province People's Hospital during 2015-2019. ESLD patients were confirmed by hepatic decompensation according to the guidelines defined by the Chinese Society of Hepatology and Chinese Society of Infectious Diseases. In the present study, patients getting anticoagulation injection showed prothrombin time (PT) change less than 5 seconds were enclosed. However, patients who were pregnant or lactating women, and who had hepatocellular carcinoma, bacterial peritonitis, human immunodeficiency virus (HIV) infection or any other life-threatening infection (e.g. hepatitis $B$ virus and hepatitis $C$ virus) recurrent gastrointestinal hemorrhage, portal thrombosis with main portal trunk obstruction, sponge blood vessel, superior mesenteric vein occlusion, or severe intestinal congestion, ascites and resistance to diuretic therapy or developed abdominal compartment syndrome, hepatic encephalopathy, and who had no complete follow-up data were not recruited into the study. The study was reviewed and approved by the Ethics Committee of Sichuan Academy of Medical 
Sciences \& Sichuan Provincial People's Hospital for stem cell research and informed consent was obtained from the patients. Written informed consents were obtained from all the participants or their legal surrogates.

\section{Preparation and treatment protocol of transplantation of UC-MSCs}

The preparation and treatment protocol of transplantation of UC-MSCs were followed by previous study (16). In detail, patients in the study groups were administered with polyene phosphatidyl choline $(0.3-0.4 \mathrm{~g} /(\mathrm{kg} \cdot \mathrm{d}))$ and magnesium isoglycyrrhizinate $(3-4 \mathrm{mg} /(\mathrm{kg} \cdot \mathrm{d}))$ for liver protection, a1-thymosin (1.6 $\mathrm{mg} / \mathrm{d}$ ) for immunity enhancement, somatostatin (24-hour continuous infusion at $250 \mathrm{mg} / \mathrm{h}$ ) and propranlolum (10-20 mg/d) for decreasing portal vein pressure. For culture of UC-MSCs, the umbilical cord was cut into $1-\mathrm{mm}^{3}$ pieces and filtered through a $1.5-\mathrm{mm}$ mesh. Filtered umbilical cord pieces were seeded in 150-cm ${ }^{2}$ flask, followed by adding DMEM/F12 complete medium. The culture medium was refreshed every 3 to $4 \mathrm{~d}$, and non-adherent cells were removed. The UC-MSCs were separated by trypsin digestion and cultured when cell confluency reached $80-90 \%$. UC-MSCs were identified and quantified by flow cytometry (FACScalibur, BD Inc., San Jose, CA, USA). UC-MSCs showed great morphology (Figure 1A). Mycoplasma contamination was assessed for UC-MSCs, and it indicated clear nucleus without fragments, which confirmed that there was no mycoplasma contamination (Figure 1B). It was indicated that UC-MSCs expressed CD105, CD73 and CD34+/CD45+ (Figure 1C). The growth curve of UNC-MSCs showed good proliferation (Figure 1D). Patients were transplanted with harvested UC-MSCs $(1.79 \pm 1.66 \times$ $10^{6}$ ) via either hepaticartery or portal vein. During transplantation procedure, the portal vein pressure was carefully assessed and monitored. A low molecular weight heparin of anticoagulant (Sanofi-Aventis, Paris, France) was treated at postoperative day 3 to prevent portal vein thrombosis.

\section{Clinical and laboratory analysis}

All patients were received the clinical examination. Moreover, patient were routinely received blood examination (hemoglobin, white blood cell count, and platelet count), liver function examination including testing ALT, AST, albumin, and total bilirubin, detection of coagulation profile including PT, aPPT, and INR, evaluation of infectious profile, blood urea, and serum creatinine levels. All patients were received ultrasound and computed tomography (CT) scan examination pre and post UC-MSCs transplantation. The liver disease severity was evaluated by using Child-Pugh score, Model of end stage liver disease (MELD) score, and indocyanine green (ICG) score. The inflammation and fibrosis were evaluated with the Ishak system. Samples used for pathological analysis was collected using liver biopsy with ultrasound or during surgery. Tissues were formalin-fixed, followed by obtaining pathological sections $(4 \mu \mathrm{m})$. Pathological sections were stained with hematoxylin and eosin (HE) and Gomori methenamine silver. All pathological results were checked and confirmed by at least two experienced pathologists. The healthrelated quality of life (QOL) of the patients was assessed using the chronic liver diseases questionnaire. 


\section{Follow-up after UC-MSCs transplantation}

All patients were followed up at 3,6,12,36, and 60 months after UC-MSCs transplantation. The results of blood test, liver function examination (ALT, AST, albumin and total bilirubin), coagulation time analyses (prothrombin time (PT) and activated partial thromboplastin time (aPPT)), immunological detection and tumor marker examinations were recorded and analyzed. The malignant tumors were ruled out using contrast-enhanced ultrasound, computed tomography (CT) scan and vascular angiography. The Knodell scoring system was used for scoring liver inflammation and fibrosis. Ascites were recorded and classified as absent (score 0), slight (score 1), moderate (score 2), and severe (score 3).

\section{Statistical Analysis}

The statistical analysis was performed using GraphPad Prism (version 5.0 for Windows). All data were presented as mean and standard deviation (SD). Comparison analysis was done repeated measures ANOVA or generalized estimating equations. Comparison analysis was done repeated measures ANOVA or generalized estimating equations. Survival analysis was performed by Kaplan-Meier method.

Univariate analysis was performed by Cox regression analysis. A $P$ value of $<0.05$ was considered to be statistically significant.

\section{Results}

\section{The clinical characteristics of non-UC-MSCs and UC-MSCs transplantation ELSD patients}

For non-UC-MSCs transplantation ELSD patients (Table 1), there were totally fifty patients including 29 males and 21 females. The mean of age was 49.86 years (range, 38-64 years). The mean body weight of the patients was $63.44 \mathrm{~kg}$. The mean body height of the patients was $163.98 \mathrm{~cm}$. The liver cirrhosis caused by hepatitis $B$ virus infection and caused by hepatitis $C$ virus infection in patients were 38 cases $(95 \%)$ and 3 cases $(7.5 \%)$, respectively. The number of patients of alcoholic cirrhosis and autoimmune cirrhosis were 8 out of $50(20 \%)$ and 1 out $50(2.5 \%)$, respectively. The number of grade A, B and C of Child-Pugh score were 18 cases, 11 cases, and 3 cases, respectively.

Table 1

Clinical characteristic of patients with Cirrhosis without UC-MSCs transplantation. 


\begin{tabular}{ll} 
Variable & Patients with Cirrhosis $(\mathrm{n}=50)$ \\
\hline Sex male $\mathrm{n}(\%)$ & $29(58 \%)$ \\
\hline Sex female $\mathrm{n}(\%)$ & $21(42 \%)$ \\
\hline Age (years) & 49.86 \\
\hline Body weight $(\mathrm{kg})$ & 63.44 \\
\hline Body height $(\mathrm{cm})$ & 163.98 \\
\hline Aetiology & \\
\hline Hepatitis B cirrhosis & $38(95 \%)$ \\
\hline Hepatitis C cirrhosis & $3(7.5 \%)$ \\
\hline Alcoholic cirrhosis & $8(20 \%)$ \\
\hline Autoimmune cirrhosis & $1(2.5 \%)$ \\
\hline Child-Pugh class & \\
\hline A (5-6) & 18 \\
\hline B (7-9) & 11 \\
\hline C ( $\geq 10)$ & 3 \\
\hline
\end{tabular}

Regarding UC-MSCs transplantation ELSD patients (Table 2), there were totally 45 patients including 35 males and 10 females. The mean of age was 48.18 years (range, 35-65 years). The mean body weight of the patients was $62.8 \mathrm{~kg}$. The mean body height of the patients was $165.84 \mathrm{~cm}$. The liver cirrhosis caused by hepatitis B virus infection in patients was 31 cases $(68.9 \%)$, and caused by hepatitis C virus infection in patients was 7 cases (15.6\%). The number of patients of alcoholic cirrhosis was 5 out of 45 (11.1\%), and the number of patients of autoimmune cirrhosis was 2 out $50(4.4 \%)$. The number of grade A of Child-Pugh score was 39 cases, the number of grade B of Child-Pugh score was 4 cases, and there were no patients to be defined as grade $\mathrm{C}$.

Table 2

Clinical characteristic of patients with Cirrhosis following UC-MSCs transplantation. 


\begin{tabular}{|ll|}
\hline Variable & Patients with Cirrhosis (n=50) \\
\hline Sex male n (\%) & $35(77.8 \%)$ \\
\hline Sex female $n(\%)$ & $10(22.2 \%)$ \\
\hline Age (years) & 48.18 \\
\hline Body weight $(\mathrm{kg})$ & 62.8 \\
\hline Body height $(\mathrm{cm})$ & 165.84 \\
\hline Aetiology & \\
\hline Hepatitis B cirrhosis & $31(68.9 \%)$ \\
\hline Hepatitis C cirrhosis & $7(15.6 \%)$ \\
\hline Alcoholic cirrhosis & $5(11.1 \%)$ \\
\hline Autoimmune cirrhosis & $2(4.4 \%)$ \\
\hline Child-Pugh class & \\
\hline A (5-6) & 39 \\
\hline B (7-9) & 4 \\
\hline C ( $\geq 10)$ & 0 \\
\hline
\end{tabular}

\section{UC-MSCs decreased incidence of the liver cancer}

To investigate effects of UC-MSCs on the incidence of the liver cancer in ELSD patients, the number of liver cancer patients in non-UC-MSCs and UC-MSCs groups was calculated. It was found that the number of liver cancer patients in non-UC-MSCs group was 6 out 50 (12\%), while the number of liver cancer patients in UC-MSCs group was 1 out 45 (2.2\%) (Figure 2). Thus, it seems that UC-MSCs reduced the incidence of liver cancer.

\section{Survival analysis}

To examine survival situation of the ELSD patients with and without UC-MSCs transplantation, the survival curves were generated. Three (two were severe jaundice and hypohepatia, one was hypoproteinemia and hepatorenal syndrome) out of 50 non-UC-MSCs transplantation patients died, while two (one was hepatorenal syndrome and multiple organ failure, and one died from hepatorenal syndrome) out of 45 UC-MSCs patients died. The liver cancer epidemiology showed gender difference in the patients (17), thus, the survival curves stratified by gender towards non-UC-MSCs and UC-MSCs transplantation ELSD patients were established. Interestingly, it indicated that the survival percentage in male patients was higher than female patients in UC-MSCs transplantation group (Figure 3A); on the 
contrary, the survival percentage in female patients was higher than male patients in non-UC-MSCs group (Figure 3B). The survival percentage in ELSD patients treated with UC-MSCs transplantation was higher than in patients treated with non-UC-MSCs transplantation (Figure 3C). So, UC-MSCs transplantation might increase survival percentage in ESLD patients.

\section{Inflammation and fibrosis scores using Ishak system and Child-Pugh class analysis for ELSD patients with and without UC-MSCs transplantation}

To evaluate the inflammation and fibrosis during follow-up period for ELSD patients with and without UCMSCs transplantation, the inflammation and fibrosis scores using Ishak system were calculated. It was shown that inflammation score decreased over the follow-up time, and inflammation score of UC-MSCs transplantation ELSD patients was lower than non-UC-MSCs transplantation patients in late follow-up period (12, 36, and 60 month) (Figure 4A). For fibrosis, it was found that the fibrosis score was decreased over the time in ELSD patients with UC-MSCs transplantation, while it was increased over the time in ELSD patients without UC-MSCs transplantation (Figure 4B). Interestingly, inflammation score in UCMSCs transplantation patients was higher than in non-UC-MSCs transplantation ELSD patients at the beginning of transplantation, while the inflammation score in UC-MSCs transplantation ELSD patients was lower than in non-UC-MSCs transplantation ELSD patients at $60^{\text {th }}$ month of follow-up. Taken together, it was plausible that UC-MSCs transplantation could relieve the liver inflammation and fibrosis compared with in ELSD patients without UC-MSCs transplantation patients.

The Child-Pugh score was one of the most indexes to assess severity of liver cirrhosis (5). To further investigate the effects of the UC-MSCs transplantation on cirrhosis in ELSD patients, a Child-Pugh class analysis was executed. It was shown that the number of patients with Child-Pugh class A was increased while the number of patients with Child-Pugh class B was decreased over the follow-up time, and the number of patients with Child-Pugh class $C$ was declined first then climbed up in non-UC-MSCs transplanted ELSD patients (Figure 5A). For UC-MSCs transplanted ELSD patients, it was indicated that the number of patients with Child-Pugh class A quickly was increased, while the number of patients with Child-Pugh class $B$ and $C$ was quickly decreased, especially, there was no patients with Child-Pugh class C after $12^{\text {nd }}$ month of follow-up (Figure $5 \mathrm{~B}$ ). Therefore, compared with non-UC-MSCs transplanted patients, UC-MSCs transplantation potently improved liver cirrhosis in ELSD patients.

\section{Complications analysis of patients with UC-MSCs transplantation}

The complications of ELSD patients treated with UC-MSCs transplantation was performed in the study. As show in Figure 6, it was found that 9 patients showed upper gastrointestinal bleeding; two patients developed into hepatorenal syndrome; two patients were observed to be hepatic encephalopathy; three 
patients developed into portal thrombosis; two patients showed hypohepatia; most of patients (28) had no serious complications. Therefore, the complication analysis of UC-MSCs transplantation indicated that this technology treating ELSD patients was relatively safe.

\section{Discussion}

It is important for discovering safe and effective therapy to treat liver cirrhosis and relieve occurrence of cirrhosis induced liver cancer. Although great advances have been obtained in treating liver cirrhosis, while high incidence of liver cancer brought by conventional therapies makes them unsatisfied to treat this disease. In the present study, we retrospectively analyzed the clinical outcomes of 50 non-UC-MSCs transplantation patients and 45 UC-MSCs transplantation patients. Compared with non-UC-MSCs transplantation, it was found that UC-MSCs transplantation could decrease incidence of liver cancer, slightly increase survival percentage. The plausibly underling mechanism is that UC-MSCs reduce Ishak inflammation and fibrosis, and improve liver cirrhosis in ELSD patients.

Untreated cirrhosis has large possibility to result in liver cancer due to long-term chronic inflammation, while improper therapeutic solutions of liver cirrhosis could not reduce risks of development of liver cancer (18). For end-stage liver cirrhosis, the liver transplantation is treated as the only definite strategy, while the limited number of donors, high costs of surgery, and requirement of long-term administration of immunosuppressive agents hamper its broad application (6). As one of new strategies treating liver cirrhosis, stem cell transplantation has been attracted more and more attention in treating this spiny disease (19). Stem cells based therapies have been confirmed to have promising effects on end-stage of liver cirrhosis by our group (20) and other groups (21). However, to the best of our knowledge, few reports were published to analyze the cancer incidence post transplantation. Thus, the present study focused on effects of UC-MSCs transplantation on liver cancer incidence in ELDS patients and possible mechanisms. Interestingly, we found that the incidence of liver cancer in ELDS patients was largely reduced by UNMSCs transplantation compared with non-UN-MSCs transplantation (Fig. 2). Similarly, UC-MSCs were found to exert certain effects on several types of cancers, for example, Han et al. found that UC-MSCs significantly inhibited proliferation of PC-3 prostate cancer cells through activation of JNK and downregulation of PI3K/AKT signaling (22). In glioma, Fan et al. found that UM-MSCs might serve as a novel cellular vehicle for delivering therapeutic molecules in therapies against glioma (23). Reduction of the liver cancer incidence may be linked to increase of the survival percentage. Indeed, it was found that UC-MSCs slightly increased survival percentage compared with non-UC-MSCs group in ELSD patients in our study (Fig. 3). Interestingly, we observed that the clinical outcomes might be related to gender (Fig. 3). There are also several studies to show that clinical characteristics after stem cell transplantation are related to gender index $(24,25)$. However, the underlying mechanism of UC-MSCs transplantation in reduction of liver cancer incidence remains unclear.

Chronic inflammation is closely related to live injury and concurrent regeneration, which may result in sequential advancement of fibrosis, cirrhosis, and finally liver cancer (26). Another unique characteristic of liver cancer is that liver cancer is greatly associated with liver fibrosis (27). The close relationship 
between liver cancer and inflammation and fibrosis provokes us to figure out the underlying mechanism of reducing liver cancer incidence of UC-MSCs. Interestingly, we found that Ishak inflammation and fibrosis scores were decreased over the follow-up time in ELSD patients with UC-MSCs transplantation, and these two parameters were lower in ELSD patients with UC-MSCs transplantation than in patients without UC-MSCs transplantation (Fig. 4). It was also demonstrated that UC-MSCs transplantation largely improved liver cirrhosis of ELSD patients (Fig. 5). Thus, it is plausible that UC-MSCs reduces incidence of liver cancer via improving liver inflammation, fibrosis and cirrhosis.

Although UC-MSCs transplantation has several benefits for liver disease patients, while the complications post transplantation should be noted. It was found that leukemia patients had great risk to suffer from early and late complications after UC-MSCs transplantation (28). In patients with refractory lupus nephritis, fever and gastrointestinal tract symptoms were common complications after UC-MSCs transplantation (29). In the present study, it was found several patients had different complications including upper gastrointestinal bleeding, hepatorenal syndrome, hepatic encephalopathy, portal thrombosis, and hypohepatia (Fig. 6). Therefore, it was proposed to diagnose these complications as early as possible for liver diseases who received UC-MSCs transplantation.

In conclusion, we found that UC-MSCs transplantation could largely decrease incidence of liver cancer, which should be linked to increase of the survival percentage. Mechanistically, we found UC-MSCs transplantation could decrease liver inflammation and fibrosis, and improve liver cirrhosis in ELSD patients compared with non-UC-MSCs transplanted patients, and this maybe associated to the UC-MSCs transplantation caused reduction of liver cancer incidence. Few complications were observed in UC-MSCs transplantation patients, which should be aware to improve survival of the patients. More efforts should be investigated towards increasing the number of patients and follow-up time to further verify the therapeutic benefits of UC-MSCs translations in treating ELSD and decreasing risks causing liver cancer.

\section{Declarations}

\section{Ethics approval and consent to patients}

The study was reviewed and approved by the Ethics Committee of Sichuan Academy of Medical Sciences \& Sichuan Provincial People's Hospital for stem cell research and informed consent was obtained from the patients. Written informed consents were obtained from all the participants or their legal surrogates.

\section{Consent for publication}

I, the undersigned, give my consent for the publication of identifiable details, which can include photograph(s) and/or videos and/or case history and/or details within the text ("Material") to be published in the above Journal and Article. I confirm that I have seen and been given the opportunity to read both the Material and the Article (as attached) to be published by the publisher. I have discussed this consent form with all authors in the study. 


\section{Availability of data and materials}

All data generated or analysed during this study are included in this published article (and its supplementary information files).

\section{Conflicts of Interest}

All authors have no conflicts of interest relevant to this article.

\section{Funding}

The study was supported by the Major research plan of Sichuan Science and Technology Department Province (grant number: 2018SZ0110), the Project of Science \& Technology Department of Sichuan Province key R \& D plan (major science and technology projects) (grant number: 2018HH0062), the Scientific research project of Sichuan Provincial Department of health (grant number: 18PJ498), the School of Medicine, University of Electronic Science and Technology of China - Sichuan Provincial People's Hospital Youth Talent (grant number: 2015QN17,2017QN08), the National Basic Research Program of China (grant number: 2015CB964703), and Chengdu City Science and Technology Bureau key R\&D support plan, technological innovation R\&D project (grant number:2019-YFYF-00131-SN).

\section{Authors' contribution}

L.L., C.L., T.F., and X.H. conceived the study, performed, and analyzed experiments; L.L., Y.Y., H.X., G.X., H.Z, L.L., G.W., and X.H. analyzed the results, wrote the first draft of manuscript and discussed the results and finally approved the manuscript; L.L., C.L., T.F., and X.H. analyzed the data and wrote the draft; all authors read and approved the final manuscript.

\section{References}

1. Peng JK, Hepgul N, Higginson IJ, Gao W. Symptom prevalence and quality of life of patients with end-stage liver disease: A systematic review and meta-analysis. Palliat Med. 2019;33:24-36.

2. Tsochatzis EA, Bosch J, Burroughs AK. Liver cirrhosis. Lancet. 2014;383:1749-61.

3. Buechler C, Haberl EM, Rein-Fischboeck L, Aslanidis C. Adipokines in Liver Cirrhosis. Int J Mol Sci 2017, 18.

4. Schwarzkopf KM, Queck A, Thomas D, Angioni C, Cai C, Freygang Y, Ruschenbaum S, Geisslinger G, Zeuzem S, Welsch C, Lange CM. Omega-3 and -6 fatty acid plasma levels are not associated with liver cirrhosis-associated systemic inflammation. PLoS One. 2019;14:e0211537.

5. Pinter M, Trauner M, Peck-Radosavljevic M, Sieghart W. Cancer and liver cirrhosis: implications on prognosis and management. ESMO Open. 2016;1:e000042. 
6. Kwak KA, Cho HJ, Yang JY, Park YS: Current Perspectives Regarding Stem Cell-Based Therapy for Liver Cirrhosis. Can J Gastroenterol Hepatol 2018, 2018:4197857.

7. Pavlov CS, Casazza G, Nikolova D, Tsochatzis E, Burroughs AK, Ivashkin VT, Gluud C. Transient elastography for diagnosis of stages of hepatic fibrosis and cirrhosis in people with alcoholic liver disease. Cochrane Database Syst Rev. 2015;1:CD010542.

8. Yin Y, Dang W, Zhou X, Xu L, Wang W, Cao W, Chen S, Su J, Cai X, Xiao S, et al. PI3K-Akt-mTOR axis sustains rotavirus infection via the 4E-BP1 mediated autophagy pathway and represents an antiviral target. Virulence. 2018;9:83-98.

9. Ahmed SO, Ghavamzadeh A, Zaidi SZ, Baldomero H, Pasquini MC, Hussain F, Alimoghaddam K, Almohareb F, Ayas M, Hamidieh A, et al. Trends of hematopoietic stem cell transplantation in the Eastern Mediterranean region, 1984-2007. Biol Blood Marrow Transplant. 2011;17:1352-61.

10. Barban JB, Simoes BP, Moraes B, Anunciacao CRD, Rocha CSD, Pintor DCQ, Guerra DC, Silva DA, Brandao ECM, Kerbauy F, et al. Brazilian Nutritional Consensus in Hematopoietic Stem Cell Transplantation: Adults. Einstein (Sao Paulo). 2020;18:AE4530.

11. Malmegrim KCR, Lima-Junior JR, Arruda LCM, de Azevedo JTC, de Oliveira GLV, Oliveira MC. Autologous Hematopoietic Stem Cell Transplantation for Autoimmune Diseases: From Mechanistic Insights to Biomarkers. Front Immunol. 2018;9:2602.

12. Peinemann F, Smith LA, Bartel C. Autologous hematopoietic stem cell transplantation following high dose chemotherapy for non-rhabdomyosarcoma soft tissue sarcomas. Cochrane Database Syst Rev 2013:CD008216.

13. Sedhom R, Sedhom D, Jaimes E. Mini-review of kidney disease following hematopoietic stem cell transplant. Clin Nephrol. 2018;89:389-402.

14. Sharrack B, Saccardi R, Alexander T, Badoglio M, Burman J, Farge D, Greco R, Jessop H, Kazmi M, Kirgizov K, et al. Autologous haematopoietic stem cell transplantation and other cellular therapy in multiple sclerosis and immune-mediated neurological diseases: updated guidelines and recommendations from the EBMT Autoimmune Diseases Working Party (ADWP) and the Joint Accreditation Committee of EBMT and ISCT (JACIE). Bone Marrow Transplant. 2020;55:283-306.

15. Copelan EA. Hematopoietic stem-cell transplantation. N Engl J Med. 2006;354:1813-26.

16. Huang XL, Luo L, Luo LY, Xue H, Wei LL, Yao YT, Zou HB, Huang XB, Zhu YF, Zhang T, et al. Clinical outcome of autologous hematopoietic stem cell infusion via hepatic artery or portal vein in patients with end-stage liver diseases. Chin Med Sci J. 2014;29:15-22.

17. An L, Zeng HM, Zheng RS, Zhang SW, Sun KX, Zou XN, Chen R, Wang SM, Gu XY, Wei WW, He J. (Liver cancer epidemiology in China, 2015). Zhonghua Zhong Liu Za Zhi. 2019;41:721-7.

18. Li J, Hu SB, Wang LY, Zhang X, Zhou X, Yang B, Li JH, Xiong J, Liu N, Li Y, et al. Autophagy-dependent generation of Axin2 + cancer stem-like cells promotes hepatocarcinogenesis in liver cirrhosis. Oncogene. 2017;36:6725-37.

19. Wang J, Sun M, Liu W, Li Y, Li M. Stem Cell-Based Therapies for Liver Diseases: An Overview and Update. Tissue Eng Regen Med. 2019;16:107-18. 
20. Luo L, Yao YT, Xue H, Luo LY, Zou HB, Wang G, Xiang GM, Wei LL, Yang MZ, Zhang T, et al. Autologous hematopoietic stem cell transplantation in patients with end-stage liver disease: a 5-year follow-up study of 48 patients. Eur Rev Med Pharmacol Sci. 2016;20:2908-15.

21. Salama H, Zekri AR, Zern M, Bahnassy A, Loutfy S, Shalaby S, Vigen C, Burke W, Mostafa M, Medhat $\mathrm{E}$, et al. Autologous hematopoietic stem cell transplantation in 48 patients with end-stage chronic liver diseases. Cell Transplant. 2010;19:1475-86.

22. Han I, Yun M, Kim EO, Kim B, Jung MH, Kim SH. Umbilical cord tissue-derived mesenchymal stem cells induce apoptosis in PC-3 prostate cancer cells through activation of JNK and downregulation of PI3K/AKT signaling. Stem Cell Res Ther. 2014;5:54.

23. Fan C, Wang D, Zhang Q, Zhou J. Migration capacity of human umbilical cord mesenchymal stem cells towards glioma in vivo. Neural Regen Res. 2013;8:2093-102.

24. Ogonek J, Kralj Juric M, Ghimire S, Varanasi PR, Holler E, Greinix H, Weissinger E. Immune Reconstitution after Allogeneic Hematopoietic Stem Cell Transplantation. Front Immunol. 2016;7:507.

25. Schulte CM, Beelen DW. Avascular osteonecrosis after allogeneic hematopoietic stem-cell transplantation: diagnosis and gender matter. Transplantation. 2004;78:1055-63.

26. Bishayee A. The role of inflammation and liver cancer. Adv Exp Med Biol. 2014;816:401-35.

27. Affo S, Yu LX, Schwabe RF. The Role of Cancer-Associated Fibroblasts and Fibrosis in Liver Cancer. Annu Rev Pathol. 2017;12:153-86.

28. Hierlmeier S, Eyrich M, Wolfl M, Schlegel PG, Wiegering V. Early and late complications following hematopoietic stem cell transplantation in pediatric patients - A retrospective analysis over 11 years. PLoS One. 2018;13:e0204914.

29. Huang X, Chen W, Ren G, Zhao L, Guo J, Gong D, Zeng C, Hu W, Liu Z. Autologous Hematopoietic Stem Cell Transplantation for Refractory Lupus Nephritis. Clin J Am Soc Nephrol. 2019;14:719-27.

\section{Figures}


A Morphology of UC-MSCs (200X)

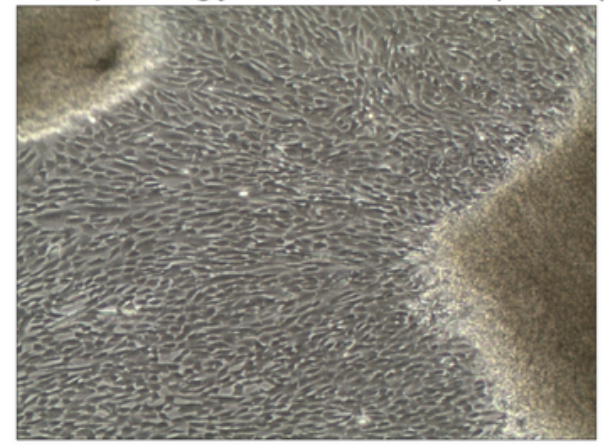

B

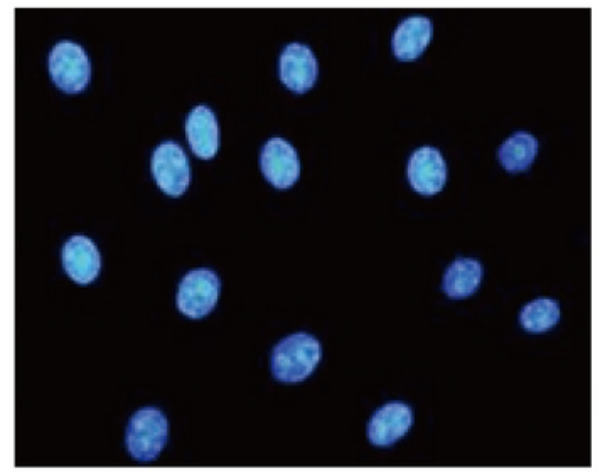

$\mathrm{C}$

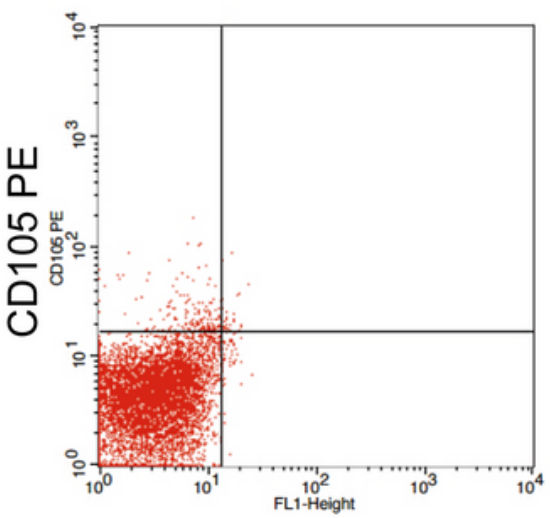

FL1-Height

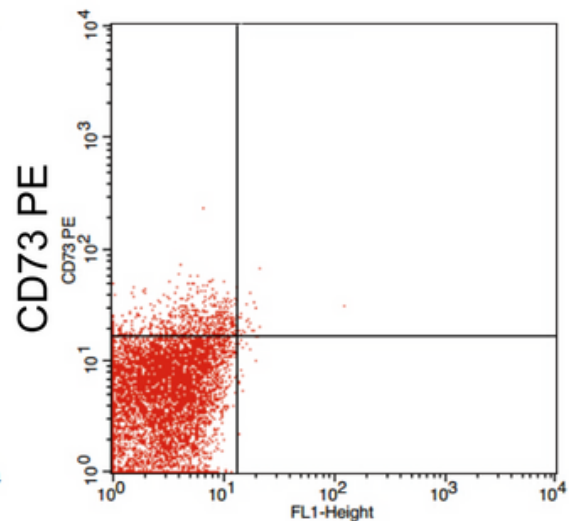

FL1-Height

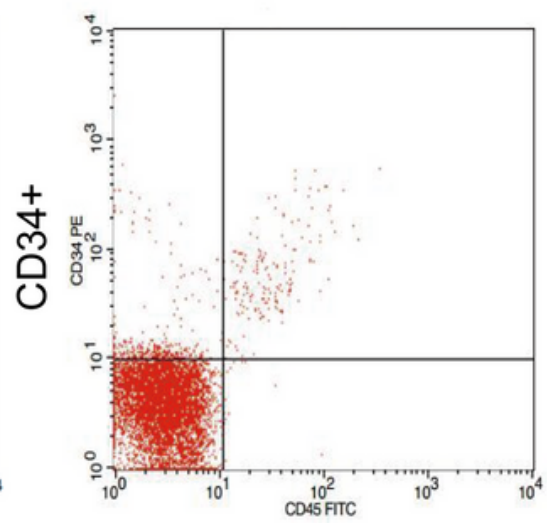

CD45+

D

\section{Growth curve of UC-MSCs}

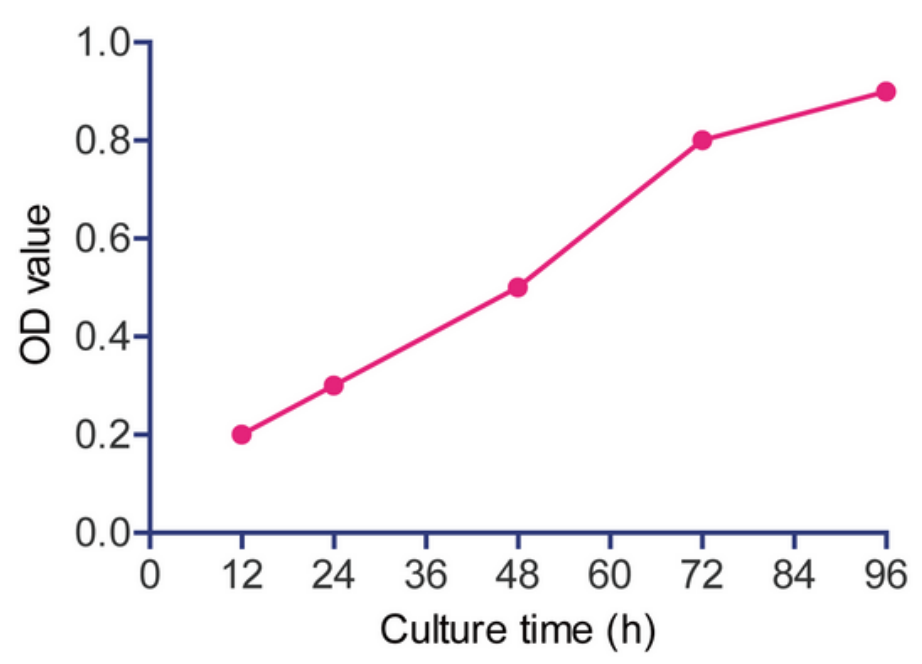

\section{Figure 1}

Culture and identification of UC-MSCs. (A) Morphology of UC-MSCs; (B) Mycoplasma contamination test for UC-MSCs; (C) FACS analysis towards cultured UC-MSCs; (D) The growth curve of UNC-MSCs. 
A

B

Hepatocarcinogenesis

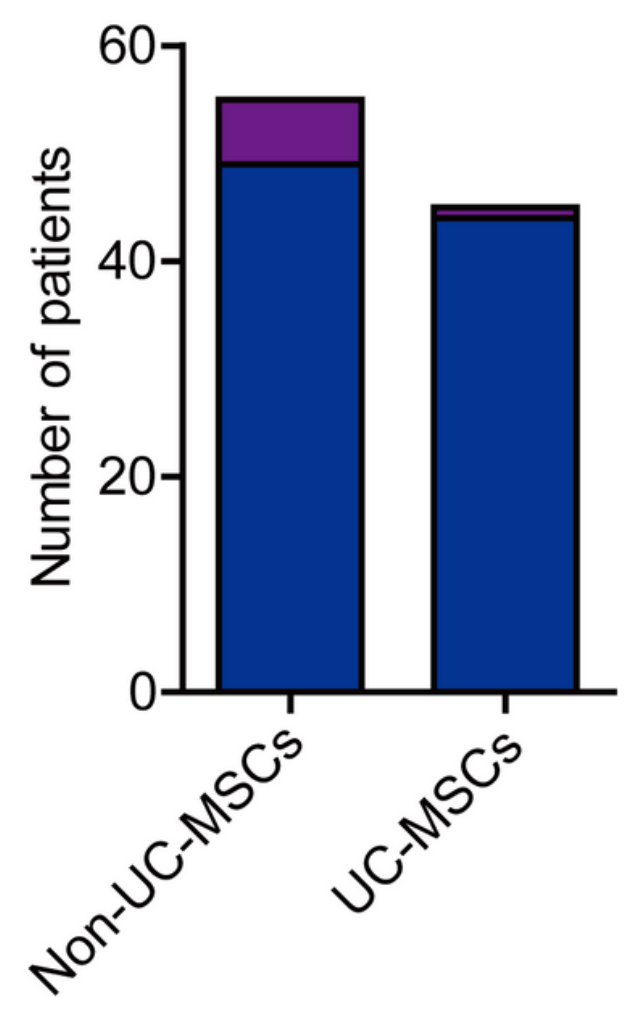

$\square$ Non-liver cancer

$\square$ Liver cancer

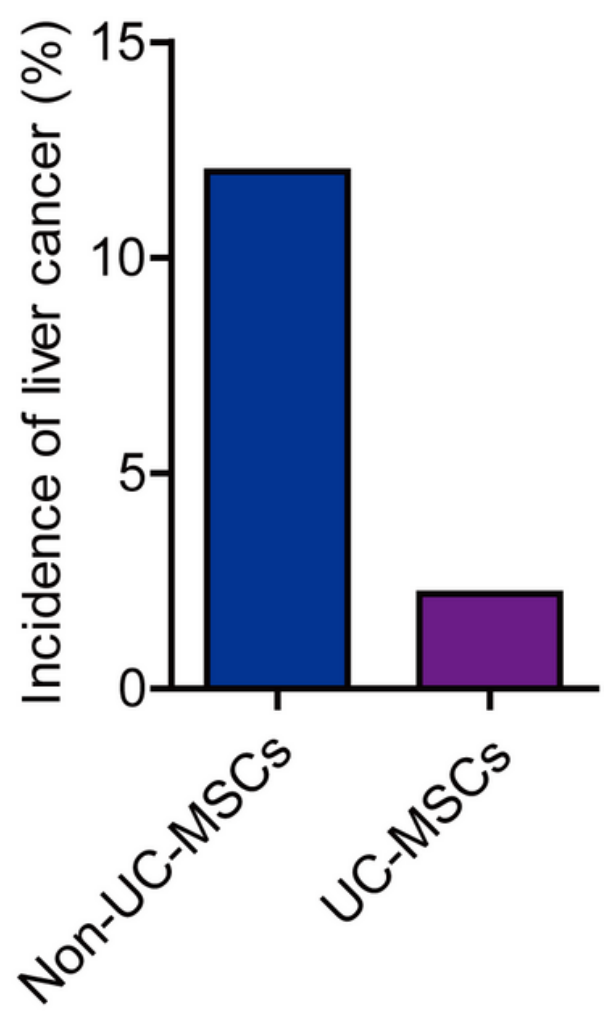

Figure 2

UC-MSCs transplantation decreased incidence of liver cancer. (A) Number of liver cancer patients in ELSD patients; (B) The incidence of liver cancer in ELSD patients. 
A

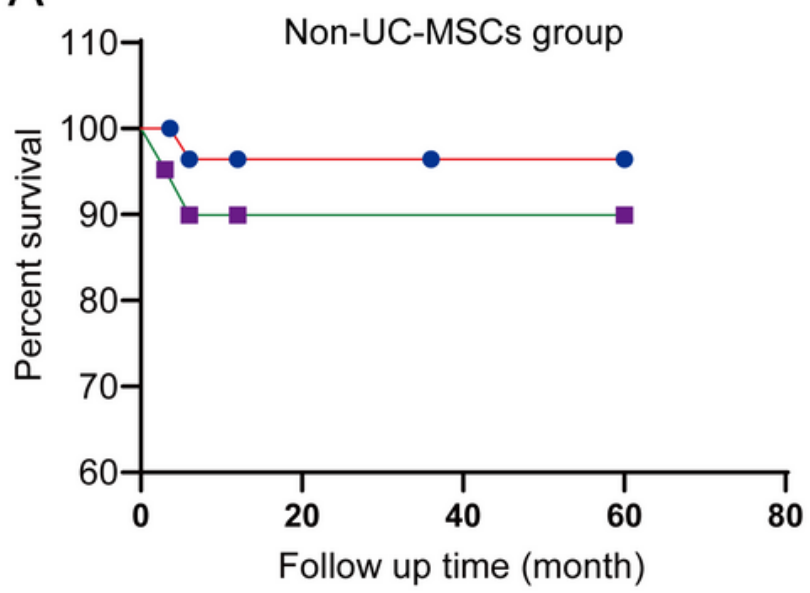

- Male

-- Female

B

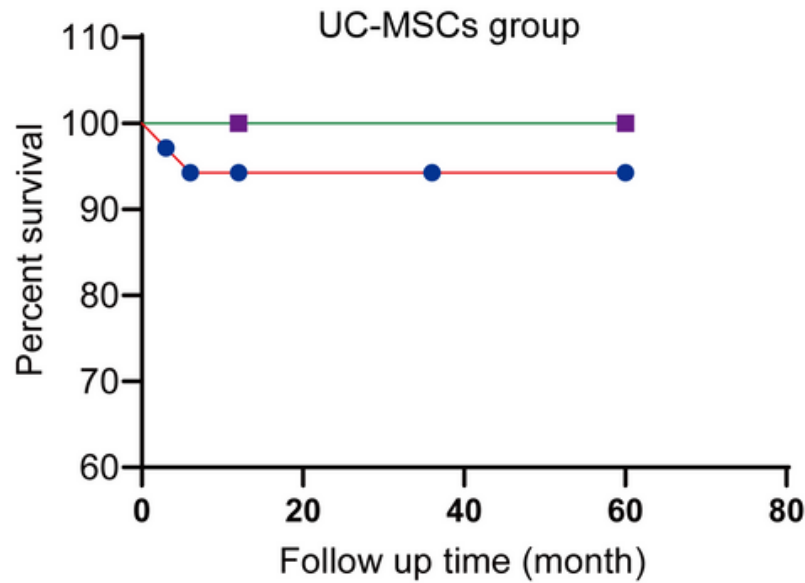

-- Male

- Female

C

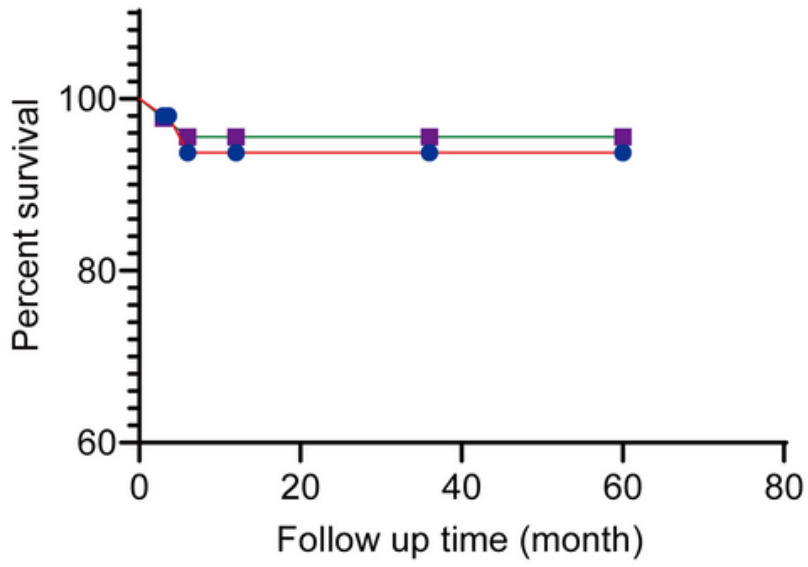

- Non-UC-MSCs group

- UC-MSCs group

\section{Figure 3}

Survival analysis of patients underwent UC-MSCs transplantation during the long term follow-up (5 years). (A) The survival curves stratified by gender of non-UC-MSCs transplanted ELSD patients; (B) The survival curves stratified by gender of UC-MSCs transplanted ELSD patients; (C) The survival percentage in patients treated with UC-MSCs transplantation was higher than in patients with UC-MSCs transplantation. 
A

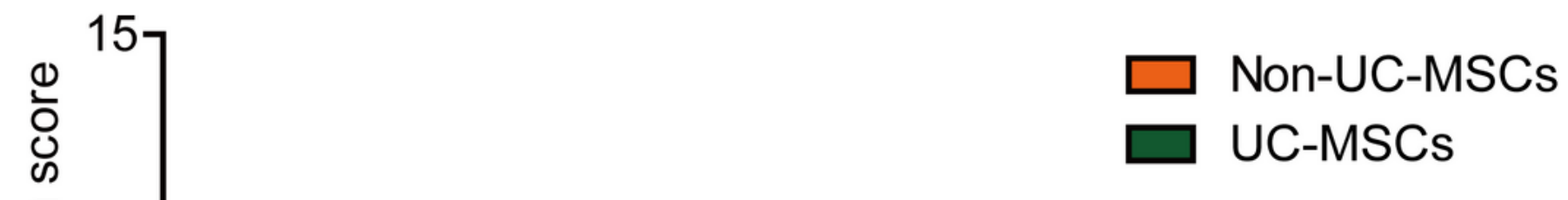

B

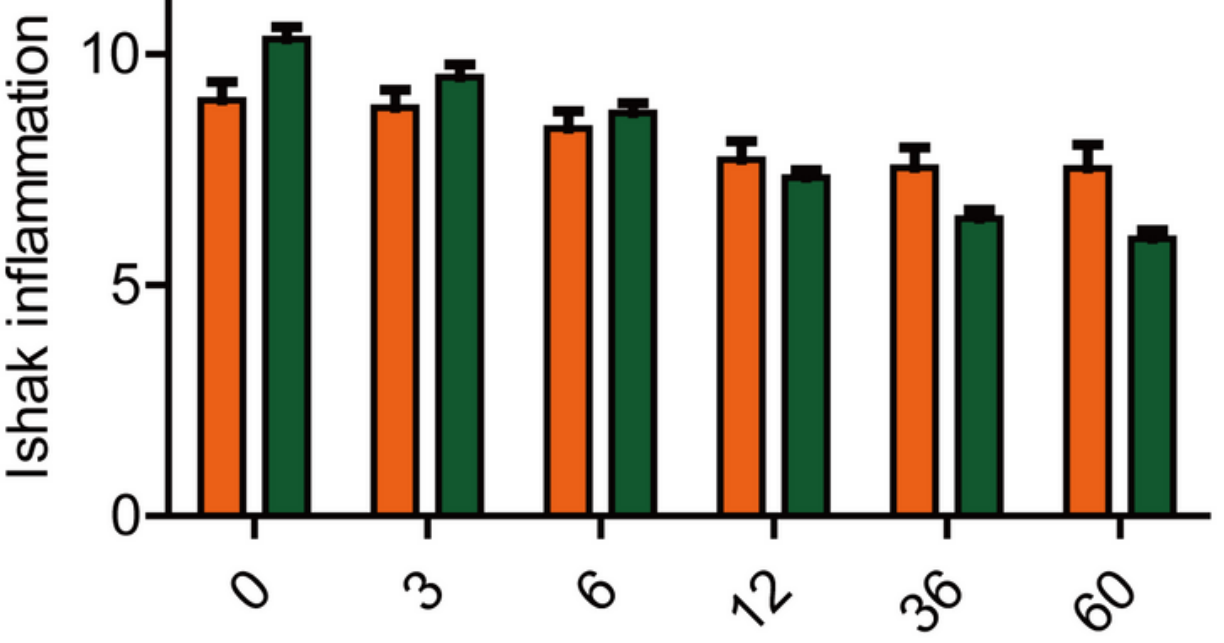

Follow up time (month)

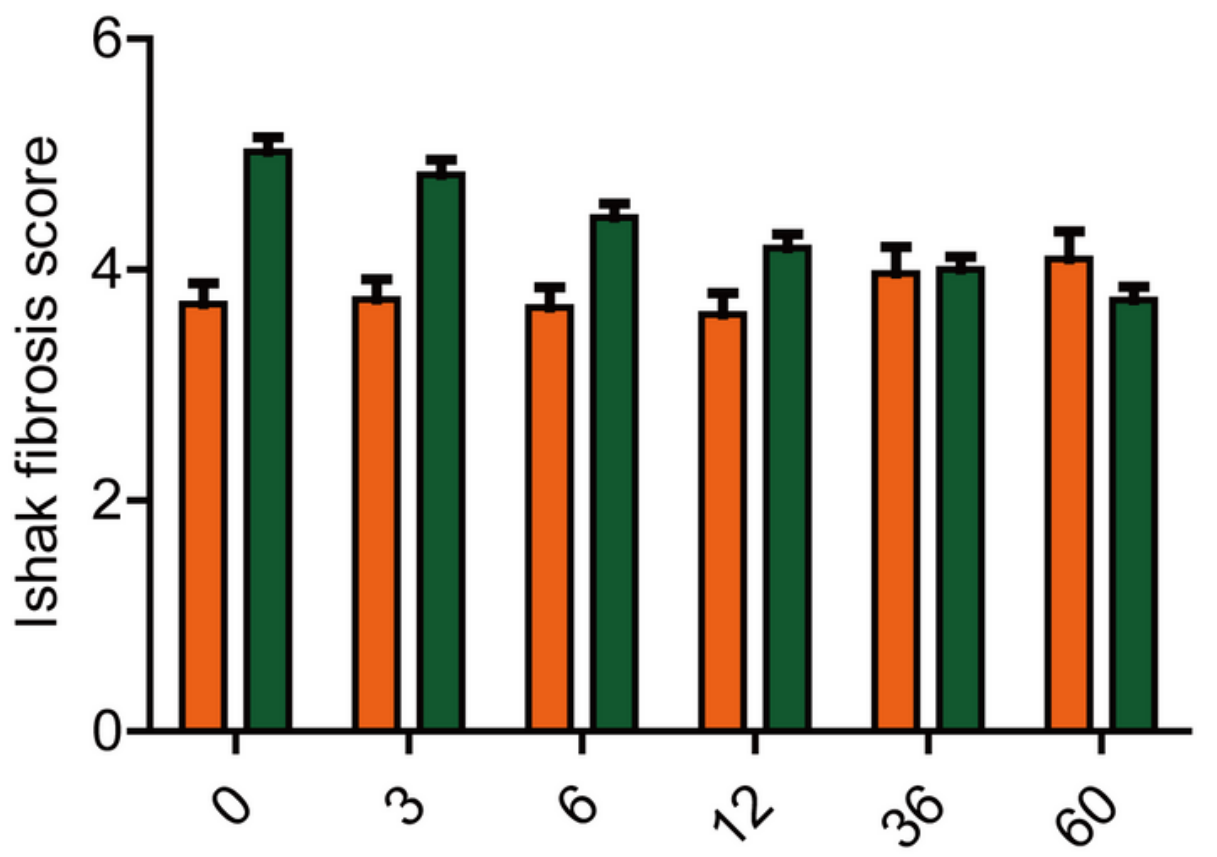

Follow up time (month)

$\square$ Non-UC-MSCs

$\square$ UC-MSCs 
A

Non-UC-MSCs transplantation

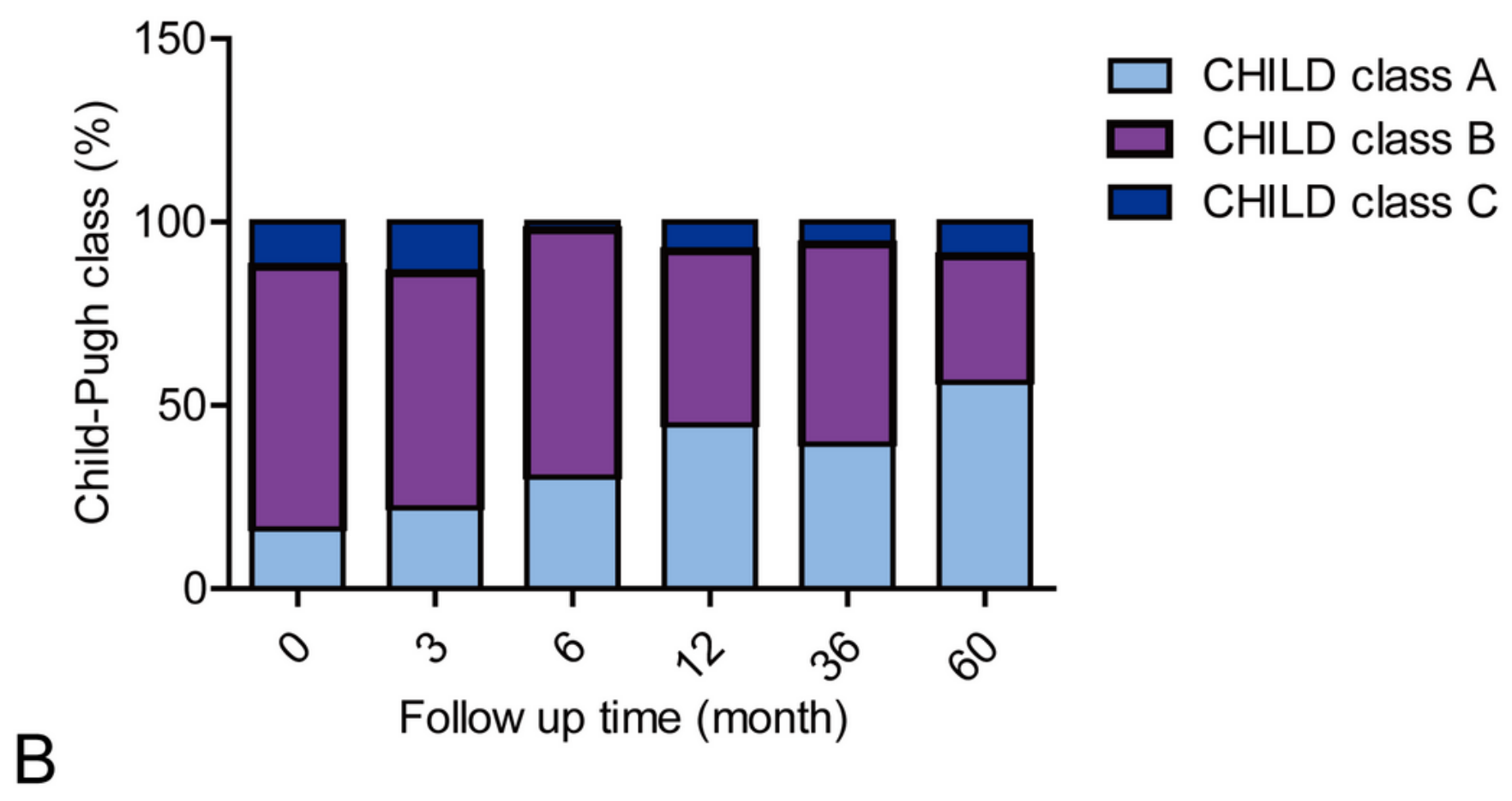

UC-MSCs transplantation

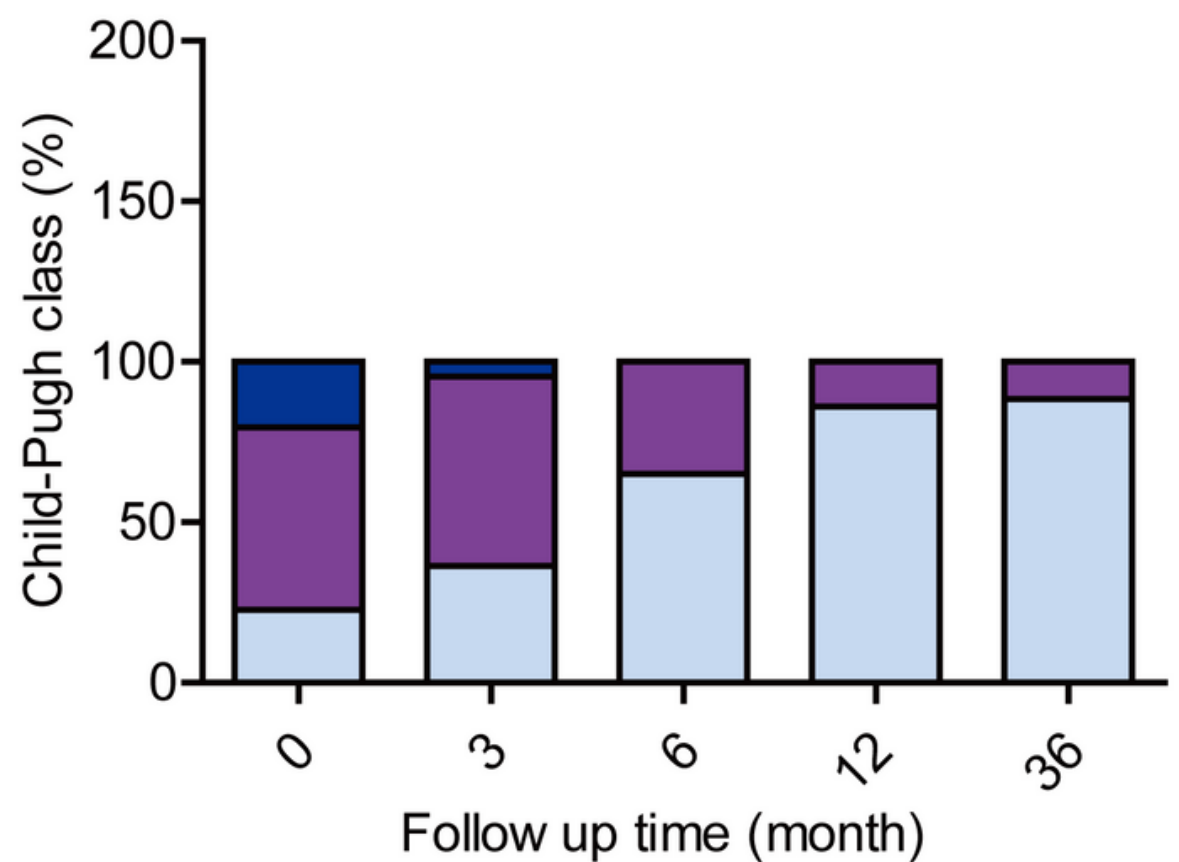

$\square$ CHILD class A $\square$ CHILD class B $\square$ CHILD class C

Figure 5

Child-Pugh classification of the ELSD patients. (A) Child-Pugh classification in non-UC-MSCs transplanted ELSD patients; (B) Child-Pugh classification in ELSD patients following UC-MSCs transplantation. 


\section{Postoperative complications during the 5 years follow-up}

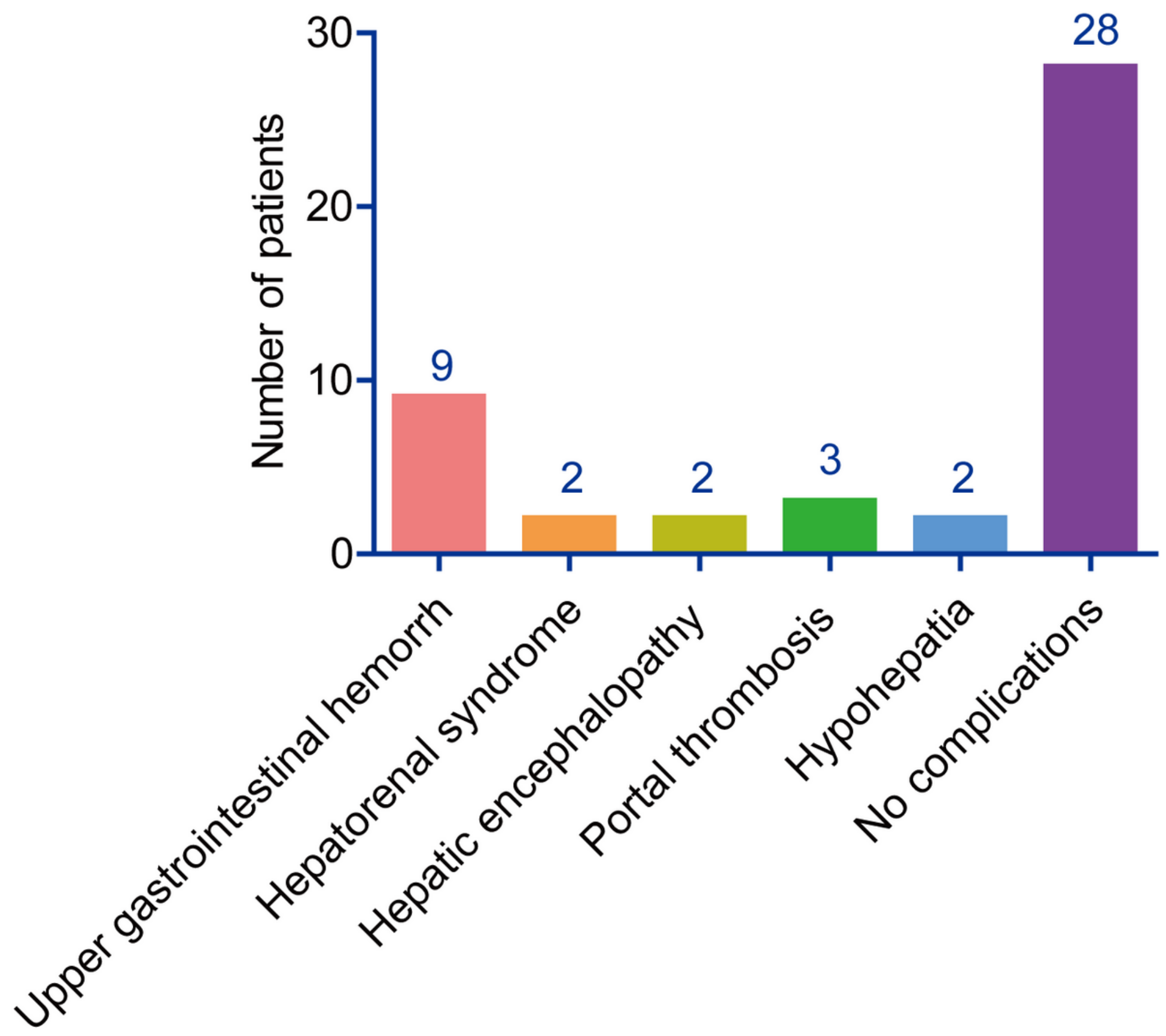

Figure 6

Postoperative complications during the 5 years follow-up in ELSD patients treated with UC-MSCs transplantation. 